\title{
A Monte Carlo simulation study of binary mixtures of charged hard spherocylinders and charged hard spheres
}

\author{
Carlos Avendaño ${ }^{\mathrm{a}, 1}$, Alejandro Gil-Villegas ${ }^{\mathrm{b}, *, 1}$, Enrique González-Tovar ${ }^{\mathrm{c}}$ \\ a Facultad de Química, Universidad de Guanajuato, Noria Alta s/n, 36050 Guanajuato, Mexico \\ b Departamento de Ingeniería Física, Campus León de la Universidad de Guanajuato, Lomas del Bosque 103, 37150 León, Guanajuato, Mexico \\ ' Instituto de Física, Universidad Autónoma de San Luis Potosí, Álvaro Obregón 64, 78000 San Luis Potosí, San Luis Potosí, Mexico
}

\section{A R T I C L E I N F O}

\section{Article history:}

Received 28 July 2008

In final form 8 January 2009

Available online 15 January 2009

\begin{abstract}
A B S T R A C T
In this work, we report Monte Carlo results for thermodynamic and structural properties of an electroneutral binary mixture of charged hard spherocylinders and charged hard spheres. We have examined the effects of the presence of charged hard spheres on the liquid crystalline phases of charged hard spherocylinders for two different models, namely, with charges located at the end or at the center of the spherocylinders. In both cases isotropic and nematic phases are observed. However, at higher densities the systems present nematic-smectic-A and nematic-columnar phase transitions for the first and second models, respectively, according to the simulated structure factor.
\end{abstract}

() 2009 Elsevier B.V. All rights reserved.

\section{Introduction}

It is well-known that rodlike colloidal particles and rodlike viruses dispersed in a solvent can exhibit liquid crystalline phases $[1,2]$. Biological and synthetic water-soluble nanotubes and nanorods are often charged and can form bundles which self-assembly in columnar liquid crystals, 2D membranes with nematic ordering, etc. $[3,4]$. A relatively simple model to describe this kind of systems consists on fluids of hard spherocylinders (HSC), which have been studied theoretically and via computer simulation by several authors [5-7].

In order to have a better understanding of the influence of some other interactions in these anisotropic materials, in a previous work we have studied a system of electroneutral charged hard spherocylinders (CHSC) particles using the NVT and NPT Monte Carlo simulation methods [8]. Coulombic interactions were managed using the Wolf method $[9,10]$ and two isotherms were analyzed and compared with those of the phase diagrams of neutral hard spherocylinders and of dipolar hard spherocylinders [6,11-19]. The liquid crystalline phases observed in the CHSC system were isotropic $(\mathrm{I})$, nematic $(\mathrm{N})$ and smectic- $\mathrm{A}(\mathrm{Sm}-\mathrm{A})$. The same phases had been detected in the dipolar and neutral HSC systems.

Due to its unquestionable relevance in diverse fields, in this Letter we present computer simulation results for a mixture of charged hard spherocylinders and charged hard spheres (CHS) employing the Monte Carlo (MC) method in the NVT ensemble.

\footnotetext{
* Corresponding author.

E-mail addresses: gil@fisica.ugto.mx, agilvm@yahoo.com.mx, agilvm@gmail.com (A. Gil-Villegas).

1 Present address: Department of Chemical Engineering, Imperial College London, South Kensington Campus, London SW7 2AZ, UK.
}

The two models considered differ in the position of the point charge within the HSC particles. The simulation of long-range Coulombic interactions was performed using the Wolf procedure, that has been proved to be as reliable as the Ewald sum method for bulk phases $[8,20]$.

\section{Simulation details}

The systems analyzed consist of mixtures of 1020 CHSC and $1020 \mathrm{CHS}$ particles. The spherocylinders have diameter $\sigma_{1}$ and an aspect ratio $L^{*}=L / \sigma_{1}=5.0$, where $L$ is the length of the cylinder; hard spheres have a diameter $\sigma_{2}$, and the ratio between the CHSC and CHS diameters was taken as $\sigma^{*}=\sigma_{1} / \sigma_{2}=2.0$. These values were chosen in order to introduce a basic asymmetry that is present in real lyotropic colloids. The valences of the species are $z_{1}$ and $z_{2}$ for CHSC and CHS, respectively. With the purpose to compare the particular effect of the coulombic interactions in the system, our simulations were performed for three different combinations of valences: $z_{1}=z_{2}=0, z_{1}=-z_{2}=1$ (also denoted as 1:1), and $z_{1}=-z_{2}=2$ (also denoted as 2:2). Two cases of the mixtures have been examined: in the first instance the charges in the CHSC particles were located at a distance $L / 2$ from the center and along the molecular axis. In the second case the charges were fixed at the centers of the CHSC particles. We will refer to these cases as the M1 and M2 models, respectively.

Scaled temperatures and CHSC packing fractions are defined by $T^{*}=k_{B} T D \sigma_{12} /\left|z_{1} z_{2}\right| e^{2}$ and $\eta=\left[(1 / 6)+\left(L^{*} / 4\right)\right] \pi N_{1} \sigma_{1}^{3} / V$, respectively, where $k_{B}$ is the Boltzmann constant, $T$ is the absolute temperature, $D$ is the dielectric constant of the surrounding media, $\sigma_{12}=\left(\sigma_{1}+\sigma_{2}\right) / 2, e$ is the protonic charge, $N_{1}$ is the number of CHSC particles, and $V$ is the volume of the system. 
The initial configuration was constructed as follows. First, the CHSC particles were placed in a face centered cubic (fcc) lattice, starting from an fcc array of hard spheres scaled in the [111] direction by the length of a spherocylinder $[6,21]$. Once this lattice was constructed, the hard spheres were located randomly in the system.

NVT Monte Carlo simulations for both models were carried out using the Wolf method to take into account the Coulombic long-range interactions. As in our previous works $[8,20]$, the values for the Wolf parameters were taken as $\alpha=4.0 / \mathscr{L}_{\min }$ and $R_{C}=0.5 \mathscr{L}_{\min }$, where $\mathscr{L}_{\min }$ is the shortest simulation box length. We have considered two different temperatures, $T^{*}=0.5$ and $T^{*}=1.0$, with CHSC packing fractions within the range $\eta=0.30-0.55$ for both models. An NVT Monte Carlo cycle consists of $N_{p}$ trial displacements (and rotations when a spherocylinder is moved) of the system, where $N_{p}$ is the total number of particles. The numbers of cycles used in this work to equilibrate the system were in the interval $1 \times 10^{6}-1.5 \times 10^{6}$ and the same numbers of cycles were employed to obtain averaged values. Additionally, for the M1 model a flip was done every cycle for a CHSC particle in order to sample more efficiently the phase space, specially at denser phases. All the displacements and rotations were adjusted in order to ensure an acceptation ratio between $30 \%$ and $50 \%$.

\section{Results}

In Table 1 the simulation results for the excess internal energy, $U^{\text {exc }} / N_{p} k_{B} T$, heat capacity at constant volume, $C_{v}^{e x c} / N_{p} k_{B}$, nematic order parameter, $S$, square root of the orientational distribution function, $g_{2}(r)$, of the CHSC in the limit of long distances (i.e., the asymptotic value at the simulation box length), and type of liquid crystalline phase are reported for the model M1 of a 1:1 mixture. For the two temperatures analyzed only isotropic, nematic and smectic-A phases were observed, similarly to that seen in the pure CHSC system [8]. The formation of the nematic phase was determined by the examination of the orientational correlation function $g_{2}(r)$ and the values of the nematic order parameter. At long distances $g_{2}(r)$ has the limit value of $S^{2}$ for nematic ordering. This condition was accomplished when $\eta=0.45$ for both temperatures (see Table 1). Clearly, those states correspond to nematic phases. On the other hand, $g_{2}(r)$ for $\eta=0.30,0.35$ and 0.40 becomes zero at larger distances, which identifies isotropic phases. When $\eta=0.50$ and 0.55 the formation of pronounced peaks in the projection of the radial distribution function of the CHSC along the director, $g_{\|}\left(r_{\|}\right)$, indicated the formation of layers associated to a smectic phase (see, for instance, Fig. 1). This occurs for both temperatures. Additionally, in Fig. 1 we present the projection of the structure factor in the perpendicular plane to the director, $S_{\perp}\left(\mathbf{q}_{\perp}\right)$ [22], the projections of the radial distribution function along and perpendicular to the director, $g_{\|}\left(r_{\|}\right)$and $g_{\perp}\left(r_{\perp}\right)$, respectively, and the snapshot of the final configuration for the state $\eta=0.55$ and $T^{*}=1.0$. The analysis of the structure factor and the short-ranged behavior of $g_{\perp}\left(r_{\perp}\right)$ indicates that the system is forming a smectic-A phase. The same effect is appreciated at $T^{*}=0.5$.

Table 1

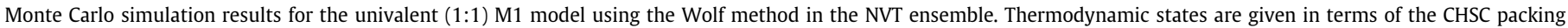

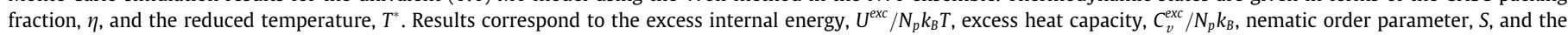

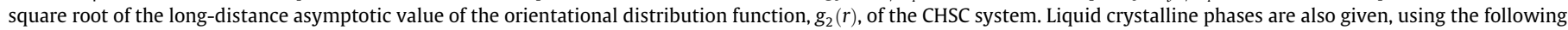
nomenclature: $\mathrm{I}=$ isotropic, $\mathrm{N}=$ nematic and $\mathrm{Sm}-\mathrm{A}=$ smectic- $\mathrm{A}$.

\begin{tabular}{|c|c|c|c|c|c|c|}
\hline$\eta$ & $T^{*}$ & $-U^{e x c} / N_{p} k_{B} T$ & $C_{v}^{e x c} / N_{p} k_{B}$ & $S$ & $\sqrt{g_{2}(\infty)}$ & Phase \\
\hline 0.30 & 1.0 & $0.256 \pm 0.006$ & $0.077 \pm 0.002$ & $0.04 \pm 0.01$ & 0.00 & I \\
\hline 0.35 & 1.0 & $0.271 \pm 0.006$ & $0.080 \pm 0.001$ & $0.06 \pm 0.02$ & 0.00 & I \\
\hline 0.40 & 1.0 & $0.286 \pm 0.006$ & $0.084 \pm 0.002$ & $0.12 \pm 0.03$ & 0.10 & I \\
\hline 0.45 & 1.0 & $0.299 \pm 0.007$ & $0.088 \pm 0.002$ & $0.79 \pm 0.01$ & 0.78 & $\mathrm{~N}$ \\
\hline 0.50 & 1.0 & $0.314 \pm 0.007$ & $0.099 \pm 0.003$ & $0.89 \pm 0.01$ & 0.88 & Sm-A \\
\hline 0.55 & 1.0 & $0.317 \pm 0.007$ & $0.111 \pm 0.003$ & $0.98 \pm 0.01$ & 0.98 & Sm-A \\
\hline 0.30 & 0.5 & $0.624 \pm 0.009$ & $0.170 \pm 0.002$ & $0.04 \pm 0.01$ & 0.00 & I \\
\hline 0.35 & 0.5 & $0.659 \pm 0.009$ & $0.175 \pm 0.002$ & $0.05 \pm 0.02$ & 0.00 & I \\
\hline 0.40 & 0.5 & $0.692 \pm 0.009$ & $0.177 \pm 0.002$ & $0.16 \pm 0.04$ & 0.14 & I \\
\hline 0.45 & 0.5 & $0.726 \pm 0.010$ & $0.187 \pm 0.004$ & $0.78 \pm 0.02$ & 0.77 & $\mathrm{~N}$ \\
\hline 0.50 & 0.5 & $0.770 \pm 0.010$ & $0.202 \pm 0.005$ & $0.91 \pm 0.01$ & 0.90 & Sm-A \\
\hline 0.55 & 0.5 & $0.791 \pm 0.010$ & $0.222 \pm 0.007$ & $0.98 \pm 0.01$ & 0.98 & Sm-A \\
\hline
\end{tabular}
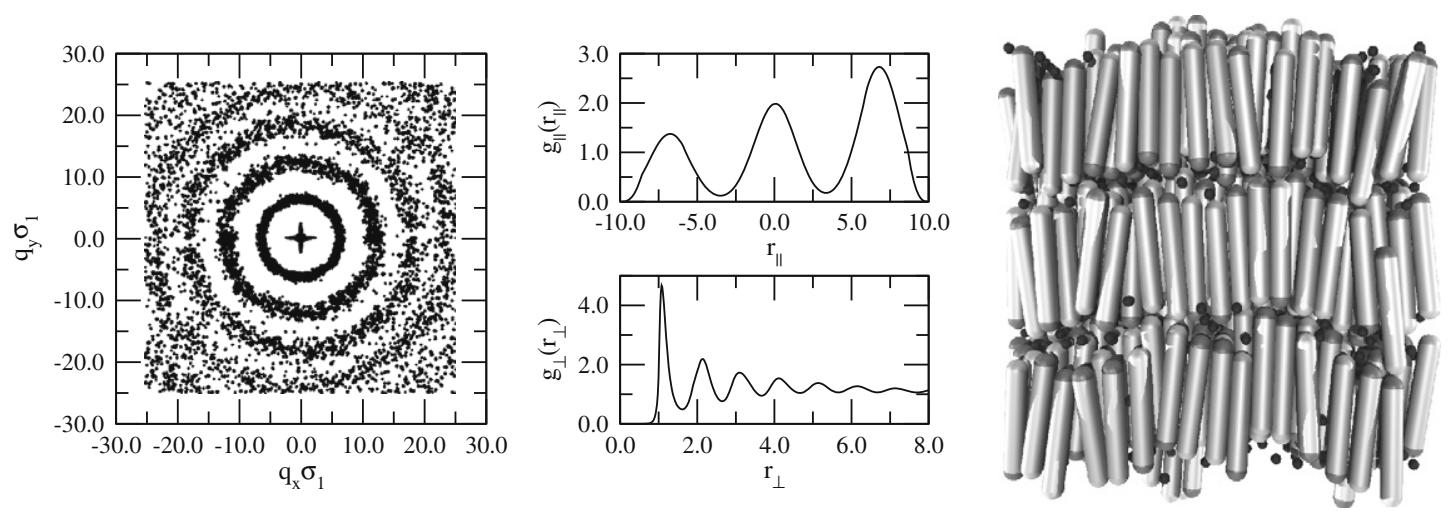

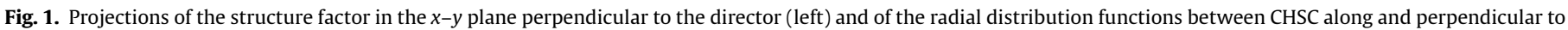

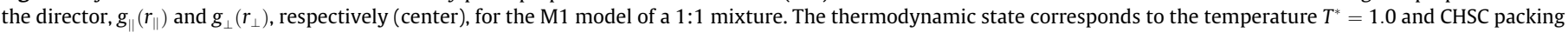
fraction $\eta=0.55$. A snapshot of the configuration of the particles is also shown (right). 
Table 2

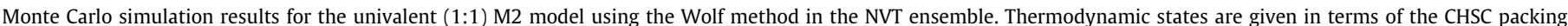

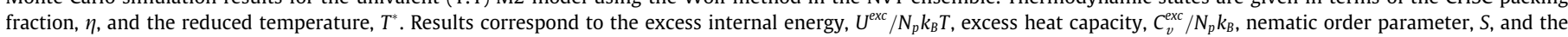

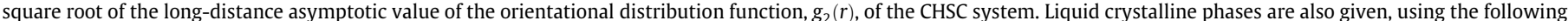
nomenclature: $\mathrm{I}=$ isotropic, $\mathrm{N}=$ nematic and $\mathrm{Col}_{\mathrm{h}}=$ hexagonal columnar.

\begin{tabular}{|c|c|c|c|c|c|c|}
\hline$\eta$ & $T^{*}$ & $-U^{e x c} / N_{p} k_{B} T$ & $C_{v}^{e x c} / N_{p} k_{B}$ & $S$ & $\sqrt{g_{2}(\infty)}$ & Phase \\
\hline 0.30 & 1.0 & $0.244 \pm 0.006$ & $0.066 \pm 0.001$ & $0.04 \pm 0.01$ & 0.00 & I \\
\hline 0.35 & 1.0 & $0.251 \pm 0.006$ & $0.074 \pm 0.001$ & $0.06 \pm 0.02$ & 0.00 & I \\
\hline 0.40 & 1.0 & $0.251 \pm 0.007$ & $0.087 \pm 0.003$ & $0.08 \pm 0.02$ & 0.00 & I \\
\hline 0.45 & 1.0 & $0.248 \pm 0.007$ & $0.097 \pm 0.005$ & $0.80 \pm 0.01$ & 0.80 & $\mathrm{~N}$ \\
\hline 0.50 & 1.0 & $0.230 \pm 0.008$ & $0.121 \pm 0.009$ & $0.92 \pm 0.01$ & 0.92 & $\mathrm{~N}$ \\
\hline 0.55 & 1.0 & $0.208 \pm 0.010$ & $0.165 \pm 0.046$ & $0.99 \pm 0.01$ & 0.99 & $\mathrm{Col}_{\mathrm{h}}$ \\
\hline 0.30 & 0.5 & $0.588 \pm 0.009$ & $0.154 \pm 0.002$ & $0.04 \pm 0.01$ & 0.00 & I \\
\hline 0.35 & 0.5 & $0.613 \pm 0.009$ & $0.168 \pm 0.002$ & $0.05 \pm 0.02$ & 0.00 & I \\
\hline 0.40 & 0.5 & $0.627 \pm 0.009$ & $0.184 \pm 0.006$ & $0.15 \pm 0.04$ & 0.14 & I \\
\hline 0.45 & 0.5 & $0.637 \pm 0.010$ & $0.203 \pm 0.006$ & $0.79 \pm 0.01$ & 0.79 & $\mathrm{~N}$ \\
\hline 0.50 & 0.5 & $0.633 \pm 0.010$ & $0.209 \pm 0.007$ & $0.92 \pm 0.01$ & 0.92 & $\mathrm{~N}$ \\
\hline 0.55 & 0.5 & $0.625 \pm 0.011$ & $0.242 \pm 0.016$ & $0.99 \pm 0.01$ & 0.99 & $\mathrm{Col}_{\mathrm{h}}$ \\
\hline
\end{tabular}

The MC-NVT results for the M2 model of 1:1 mixtures are reported in Table 2. For this case and the same temperatures used for the already discussed M1 system, the liquid crystalline phases found were isotropic, nematic and hexagonal columnar $\left(\mathrm{Col}_{h}\right)$. The occurrence of the nematic phase for this model was characterized in similar way as in the previous instance M1. For $T^{*}=1.0$ and
$T^{*}=0.5$ the nematic phase was observed at $\eta=0.45$ and 0.50 . For $\eta=0.30,0.35$ and 0.40 the phase was isotropic due to the lack of long-ranged order in $g_{2}(r)$. As can be seen in Fig. 2 for $\eta=0.55$, the formation of peaks in the columnar distribution function, $g_{c}\left(r_{\|}\right)$, can be explained by the formation of columns in the system. These columns are arranged in hexagonal order in the plane
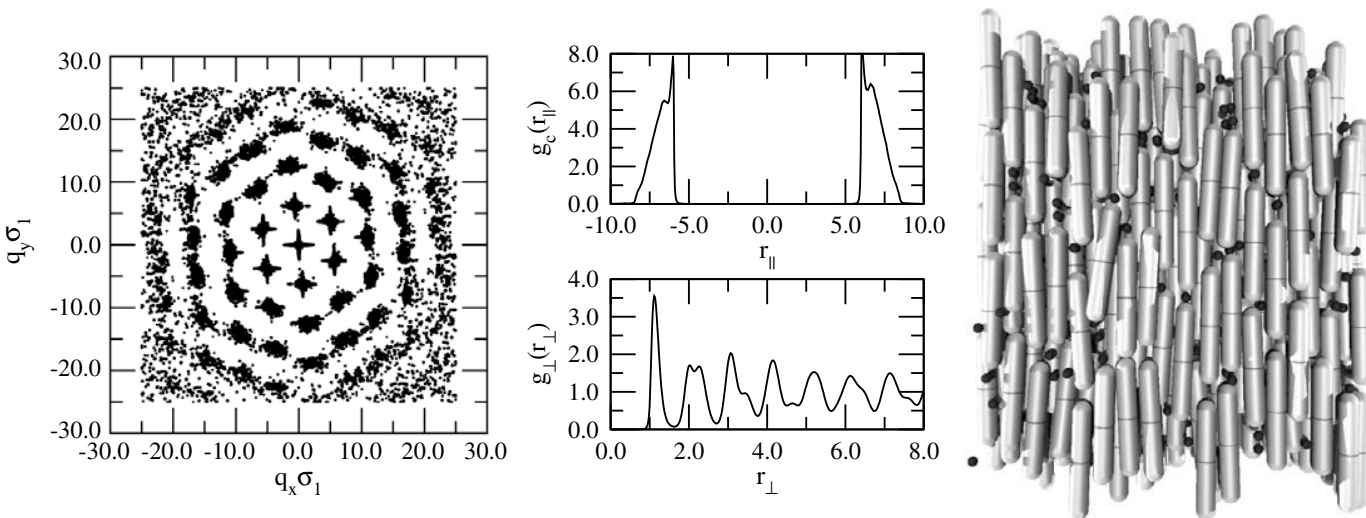

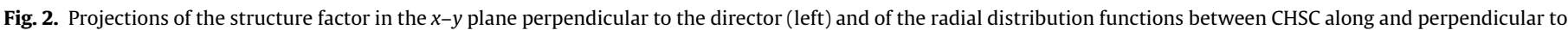

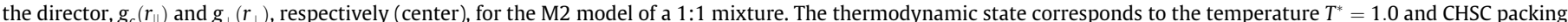
fraction $\eta=0.55$. A snapshot of the configuration of the particles is also shown (right).
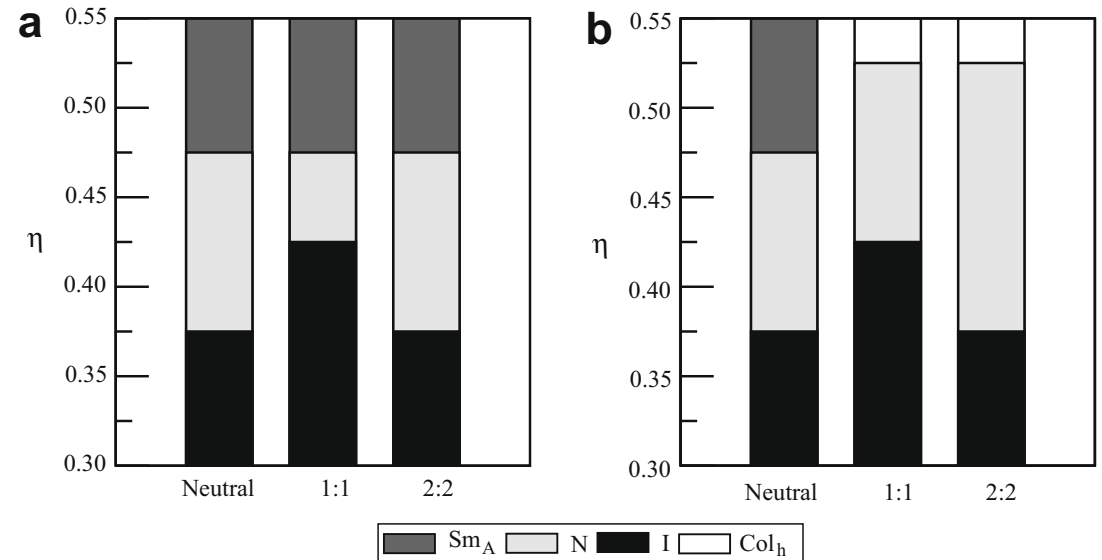

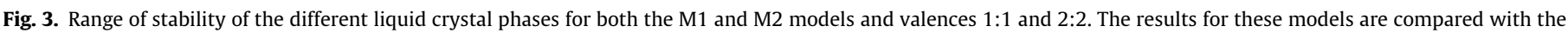
uncharged mixture. 

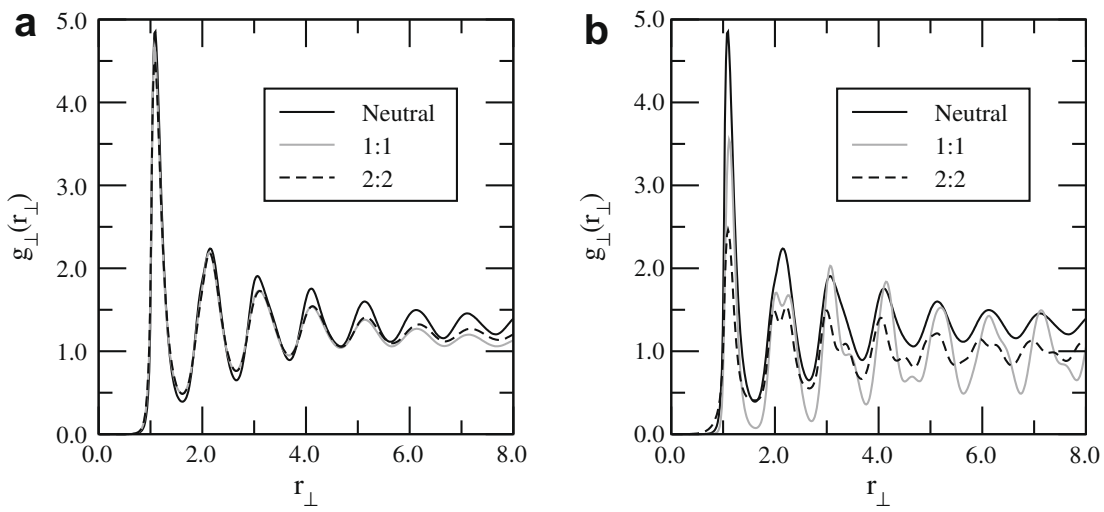

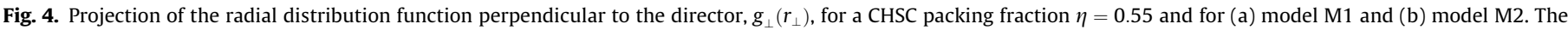
results of both models for charged systems are compared with the distribution function of the uncharged mixture.

perpendicular to the director, as portrayed in Fig. 2 where we present the structure factor projected onto the perpendicular plane to the director.

In order to know if the formation of the columnar phase is induced by coulombic forces, in addition to the 1:1 systems, simulations were performed for neutral and 2:2 mixtures. The ranges of stability for both the M1 and M2 models with valences 1:1 and 2:2 are presented in Fig. 3 and contrasted with the neutral system. As it can be observed, M1 presents the same liquid crystal structures than the neutral system, i.e., isotropic, nematic and smectic-A phases. The same phases for several uncharged mixtures of HSC and HS have been reported by other authors [23,24]. As an example of the ordering that can be observed in this instance at high densities, the distribution function $g_{\perp}\left(r_{\perp}\right)$ for $\eta=0.55$ for model M1 and different valences is shown in Fig. 4a, including the neutral system. The three functions have liquid-like behavior as found in the Sm-A phase. On the other hand, in model M2 different structures can be detected from $g_{\perp}\left(r_{\perp}\right)$, going from Sm-A phase for the neutral system to $\mathrm{Col}_{\mathrm{h}}$ phase for the charged system, where long-range order is observed (see Fig. 4b). Then it is clear that the intensity of the charge and its position are the driven forces that stabilize the columnar phase.

\section{Conclusions}

In this work, Monte Carlo results for thermodynamic and structural properties of two different models (M1 and M2) of an electroneutral binary mixture of charged hard spherocylinders and charged hard spheres have been reported. Both M1 and M2 systems differ in the position of the puntual charge along the main axis of the CHSC particles. Isotropic and nematic phases are observed for the two systems. However, at larger packing fractions the location of the charges in the CHSC has a crucial effect in the phase diagram, since the phase observed for the M1 system is a smectic-A, whereas for the M2 system is an hexagonal columnar.

When the charge is placed at the center, the configurational energy is minimized when the CHS particles are near to the center of the spherocylinders. This prevents that at high densities the centers of the spherocylinders can be aligned perpendicularly to the molecular axes to form the smectic phase. The arrangement in columns in an hexagonal order in this model is energetically most favorable. On the other hand, when the charge is placed at the end of the cylinder, the centers of spherocylinders can be aligned to form the Sm-A phase with CHS particles located between the layers.

The behavior of these systems at high values of the packing fraction is of great interest for some bio-macromolecular systems, such as tobacco mosaic virus, where a crossover from smectic order to a columnar or three dimensional crystalline order has been observed [25]. Recently, Wensink [26] has studied the stability of inhomogeneous liquid crystalline states of monodisperse, stiff charged rods using a bifurcation analysis of the Onsager free energy for charged rods in strongly nematic phases and has found that the nematic-smectic transition preempts the nematic-columnar one in the regime of strong screening, however a stable hexagonal columnar order can be observed at larger screening lengths.

Another area of interest for the present results is the formation of 2D periodic structures in holographic polymer-dispersed liquid crystal materials because of their advanced electro-optic switching capability [27]. The simulation data reported here suggest that, by controlling the location of the charge distribution in rigid polyelectrolytes, it could be also possible to induce 2D hexagonal arrays for their use in electrically switchable hexagonal photonic crystals.

\section{Acknowledgements}

We thank CONACYT (Consejo Nacional de Ciencia y Tecnología, México) for financial support (grants 58470, 61418), for a $\mathrm{PhD}$ scholarship (C.A.), and for a sabbatical leave (A.G.V). The authors would like to thank Miguel Angel Ojeda-López (Instituto de Física, Universidad Autónoma de San Luis Potosí, México) and Antonio Muñoz-Flores (Departamento de Física, Universidad Autónoma Metropolitana Iztapalapa, México) for useful discussions and suggestions.

\section{References}

[1] H. Maeda, Y. Maeda, Phys. Rev. Lett. 90 (2003) 018303.

[2] D. van der Beek, H.N.W. Lekkerkerker, Langmuir 20 (2004) 8582

[3] D.J. Needleman, M. Ojeda-López, U. Raviv, H.P. Miller, L. Wilson, C.R. Safinya, PNAS 101 (2004) 16099.

[4] G. Wong, Curr. Opin. Colloid Interf. Sci. 11 (2006) 310.

[5] M.P. Allen, G.T. Evans, D. Frenkel, B.M. Mulder, Adv. Chem. Phys. 86 (1993) 1

[6] S.C. McGrother, D.C. Williamson, G. Jackson, J. Chem. Phys. 104 (1996) 6755.

[7] P. Bolhuis, D. Frenkel, J. Chem. Phys. 106 (1997) 666.

[8] C. Avendaño, A. Gil-Villegas, E. González-Tovar, J. Chem. Phys. 128 (2008) 044506.

[9] D. Wolf, Phys. Rev. Lett. 68 (1992) 3315.

[10] D. Wolf, P. Keblinski, S.R. Phillpot, J. Eggebrecht, J. Chem. Phys. 110 (1999) 8254.

[11] J.J. Weis, D. Levesque, G.J. Zarragoicoechea, Phys. Rev. Lett. 69 (1992) 913

[12] D. Levesque, J.J. Weis, G.J. Zarragoicoechea, Phys. Rev. E 47 (1993) 496

[13] J.J. Weis, D. Levesque, G.J. Zarragoicoechea, Mol. Phys. 80 (1993) 1077.

[14] S.C. McGrother, A. Gil-Villegas, G. Jackson, J. Phys.: Condens. Matter 8 (1996) 9649.

[15] A. Gil-Villegas, S.C. McGrother, G. Jackson, Mol. Phys. 92 (1997) 723.

[16] A. Gil-Villegas, S.C. McGrother, G. Jackson, Chem. Phys. Lett. 269 (1997) 441.

[17] S.C. McGrother, A. Gil-Villegas, G. Jackson, Mol. Phys. 95 (1998) 657.

[18] A. Gil-Villegas, G. Jackson, S.C. McGrother, J. Mol. Liq. 76 (1998) 171. 
[19] J.S. van Duijneveldt, A. Gil-Villegas, G. Jackson, M.P. Allen, J. Chem. Phys. 112 (2000) 9092.

[20] C. Avendaño, A. Gil-Villegas, Mol. Phys. 104 (2006) 1475.

[21] D. Frenkel, J. Phys. Chem. 92 (1988) 3280.

[22] M. Bates, G.R. Luckhurst, J. Chem. Phys. 118 (2003) 6605.

[23] S. Lago, A. Cuetos, B. Martínez-Haya, L.F. Rull, J. Mol. Recognit. 17 (2004) 417.
[24] A. Cuetos, B. Martínez-Haya, S. Lago, L.F. Rull, Phys. Rev. E 75 (2007) 061701.

[25] S. Fraden, Observation Prediction and Simulation of Phase Transitions in Complex Fluids, Kluwer, Dordrecht, 1995.

[26] H.H. Wensink, J. Chem. Phys. 126 (2007) 194901.

[27] X. Sun, X. Tao, T. Ye, P. Sue, Y. Szeto, Appl. Phys. B 87 (2007) 267. 